\title{
Silencing of the Rice Gene LRR1 Compromises Rice Xa21 Transcript Accumulation and XA21-Mediated Immunity
}

\author{
Daniel F. Caddell ${ }^{1}$, Chang-Jin Park ${ }^{1,2}$, Nicholas C. Thomas ${ }^{1,3}$, Patrick E. Canlas ${ }^{1}$ and Pamela C. Ronald ${ }^{1 *}$
}

\begin{abstract}
Background: The rice immune receptor XA21 confers resistance to Xanthomonas oryzae pv. oryzae (Xoo), the causal agent of bacterial leaf blight. We previously demonstrated that an auxilin-like protein, XA21 BINDING PROTEIN 21 (XB21), positively regulates resistance to Xoo.

Results: To further investigate the function of XB21, we performed a yeast two-hybrid screen. We identified 22 unique XB21 interacting proteins, including LEUCINE-RICH REPEAT PROTEIN 1 (LRR1), which we selected for further analysis. Silencing of LRR1 in the XA21 genetic background (XA21-LRR1Ri) compromises resistance to Xoo compared with control XA21 plants. XA21-LRR1Ri plants have reduced Xa21 transcript levels and reduced expression of genes that serve as markers of XA21-mediated activation. Overexpression of $L R R 1$ is insufficient to alter resistance to Xoo in rice lines lacking XA21.
\end{abstract}

Conclusions: Taken together, our results indicate that LRR1 is required for wild-type Xa21 transcript expression and XA21-mediated immunity.

Keywords: Rice, Immunity, Xanthomonas oryzae pv. oryzae, XA21, XB21, LRR1, Leucine rich repeat

\section{Background}

Cell surface immune receptors, which often perceive conserved microbial signatures present outside host cells, represent a first line of defense against potential pathogens. Well characterized plant cell surface immune receptors include Arabidopsis FLAGELLIN SENSING 2 (FLS2), which recognizes a 22 amino acid epitope derived from bacterial flagellin (flg22) (Boller and Felix 2009), EF-TU RECEPTOR (EFR) which recognizes an 18 amino acid epitope derived from bacterial elongation factor-Tu (elf18) (Zipfel et al. 2006), and rice XA21, which recognizes a 21 amino acid tyrosine-sulfated epitope derived from the bacterial protein REQUIRED FOR ACTIVATION OF XA21-MEDIATED IMMUNITY X (RaxX; RaxX21-sY) (Pruitt et al. 2015). These

\footnotetext{
* Correspondence: pcronald@ucdavis.edu

${ }^{1}$ Department of Plant Pathology and the Genome Center, University of California, Davis, Davis, CA 95616, USA

Full list of author information is available at the end of the article
}

immune receptors fall into the leucine-rich repeat receptor-like kinase class (LRR-RLK) consisting of an Nterminal signal peptide, predicted extracellular LRR domain, transmembrane domain and an intracellular kinase domain. Upon microbial recognition, the kinase domain is hypothesized to initiate a signaling cascade that leads to resistance against specific pathogens. These immune receptors require the presence of LRR-RLK coreceptors that are part of the SOMATIC EMBRYOGENESIS RECEPTOR KINASE (SERK) family. For example, FLS2-mediated immune response requires the BRI1ASSOCIATED RECEPTOR KINASE (BAK1) (Chinchilla et al. 2007) and XA21-mediated immune response requires OsSERK2 (Chen et al. 2014).

Despite the importance of immune receptors to plant survival, few components that mediate the downstream signaling cascade have yet been characterized. One method that has been successful at identifying such proteins is the yeast two-hybrid system. We used this 
approach to generate an XA21 interactome (Seo et al. 2011). One of the isolated proteins was named XA21 BINDING PROTEIN 21 (XB21). XB21 encodes an auxilin-like protein that regulates resistance to $X o o$ and is predicted to function in clatharin-mediated endocytosis (Park et al. manuscript in preparation). To investigate the function of XB21, we performed a yeast two-hybrid screen utilizing XB21 as bait with a rice cDNA library. From this analysis, we identified LEUCINE RICH REPEAT PROTEIN 1 (LRR1). LRR1 shares 55\% identity and $68 \%$ similarity with the extracellular domain of OsSERK2. To determine if LRR1 is required for XA21mediated immunity, we assessed its function in rice plants in the presence and absence of XA21.

Transgenic rice plants expressing $\mathrm{Xa21}$ that were silenced for LRR1 (XA21-LRR1Ri) displayed enhanced susceptibility to Xoo. XA21-LRR1Ri plants also have decreased expression of Xa21 transcripts. These results demonstrate that LRR1 is required for XA21 -mediated immune responses and for $\mathrm{Xa21}$ transcription. These results also highlight the diverse roles played by LRR containing proteins in regulating plant responses against pathogens.

\section{Results}

LRR1 was Identified Through a Yeast two-Hybrid Screen to Identify Components of XA21-Mediated Immunity

Because full-length XB21 displayed auto-activity in the yeast two-hybrid system, an N-terminal deleted XB21 $(\mathrm{XB} 21 \Delta \mathrm{NT})$ was expressed in yeast and used as bait to screen a rice cDNA library pool derived from leaf mRNA of 7-week old rice plants containing Xa21 (Seo et al. 2011). This screen identified 22 unique rice gene IDs that we named XB21-INTERACTING PROTEINS (XB21IPs) (Table 1). We isolated two independent clones (clone 3 and 16) that encoded LRR1 (Os01g59440). To validate the interaction between XB21 and LRR1, HisXB21 was co-incubated with GST or GST-LRR1 that were purified from E. coli. His-XB21 was pulled down with GST-LRR1, but not GST, indicating that LRR1 and XB21 interact in vitro (Additional file 1: Figure S1). We then selected LRR1 for further characterization due to its similarity with OsSERK2 (Fig. 1), which is a required component of XA21-mediated immunity (Chen et al. 2014). LRR 1 and OsSERK 2 share 55\% identity and 68\% similarity over the region spanning the length of LRR1 (Kearse et al. 2012). LRR1 is predicted to encode a 213 amino acid protein containing an $\mathrm{N}$-terminal signal peptide (Amino acids 1-21), N-terminal LRR capping domain (AA 24-63) (Kolade et al. 2006), and five consecutive leucine-rich repeats (AA 65-184) following the plantspecific LRR consensus sequence LxxLxxLxLxxNxLSGx IPxxLGx (McAndrew et al. 2014) (Fig. 1). LRR1 is predicted to have two $\mathrm{N}$-glycosylation sites compared to six in the
Table 1 List of identified XB21 Interacting Proteins (XB21IPs)

\begin{tabular}{|c|c|c|}
\hline Clone ID & MSU Annotation ${ }^{a}$ & Locus ID \\
\hline 1 & $\begin{array}{l}\text { NAD dependent epimerase/dehydratase } \\
\text { family protein }\end{array}$ & LOC_Os03g23980 \\
\hline 2 & thioredoxin & LOC_Os12g08730 \\
\hline 3 & BRI1-associated kinase 1 precursor & LOC_Os01g59440 \\
\hline 4 & $\begin{array}{l}\text { NAD dependent epimerase/dehydratase } \\
\text { family protein }\end{array}$ & LOC_Os03g23980 \\
\hline 5 & Glutaminyl-tRNA synthetase & LOC_Os01g09000 \\
\hline 6 & Beta-D-glucan exohydrolase-like protein & LOC_Os05g37700 \\
\hline 7 & FHA domain containing protein & LOC_Os11g03390 \\
\hline 8 & $\begin{array}{l}\text { OsCML24 - Calmodulin-related calcium } \\
\text { sensor protein }\end{array}$ & LOC_Os07g48340 \\
\hline 9 & metallothionein-like protein & LOC_Os01g10400 \\
\hline 10 & peptidyl-tRNA hydrolase & LOC_Os01g49900 \\
\hline 11 & photosystem I reaction center subunit & LOC_Os07g05480 \\
\hline 12 & phosphoesterase family protein & LOC_Os03g61130 \\
\hline 13 & peroxiredoxin & LOC_Os02g33450 \\
\hline 14 & dehydrin & LOC_Os03g45280 \\
\hline 15 & $\begin{array}{l}\text { universal stress protein domain } \\
\text { containing protein }\end{array}$ & LOC_Os07g36600 \\
\hline 16 & BRI1-associated kinase 1 precursor & LOC_Os01g59440 \\
\hline 17 & glutamine synthetase & LOC_Os02g50240 \\
\hline 18 & ZIM domain containing protein & LOC_Os10g25290 \\
\hline 19 & transmembrane protein 56 & LOC_Os03g46410 \\
\hline 20 & AAA-type ATPase family protein & LOC_Os11g47970 \\
\hline 21 & WRKY53 & LOC_Os05g27730 \\
\hline 22 & $\mathrm{ABC} 1$ family domain containing protein & LOC_Os07g12530 \\
\hline 23 & $\begin{array}{l}\text { bZIP transcription factor domain } \\
\text { containing protein }\end{array}$ & LOC_Os01g58760 \\
\hline 24 & metallothionein-like protein & LOC_Os01g10400 \\
\hline 25 & Ser/Thr receptor-like kinase & LOC_Os01g02560 \\
\hline
\end{tabular}

${ }^{a}$ MSU Isa1 Release 7 (http://rice.plantbiology.msu.edu/)

OsSERK2 ectodomain (Zhang et al. 2004). Unlike OsS ERK2, LRR1 does not contain a transmembrane domain or intracellular domain.

\section{Silencing of LRR1 in the Xa21 Genetic Background Enhances Susceptibility to Xoo}

To investigate the role of LRR1 in XA21-mediated immunity, RNAi was used to study the effects of LRR1 silencing in vivo. Kitaake plants carrying $\mathrm{Xa21}$ with its native promoter, XA21 23A-1-14 (referred to as XA21 rice plants in this paper) (Park et al. 2010), were transformed with the LRR1-RNAi (LRR1Ri) construct by Agrobacterium-mediated plant transformation. Five independent XA21-LRR1Ri $\mathrm{T}_{0}$ lines were inoculated with Xoo strain PXO99Az (PXO99). Independently transformed lines 1, 3, and 4 showed an enhanced susceptibility phenotype and were advanced to the next generation 


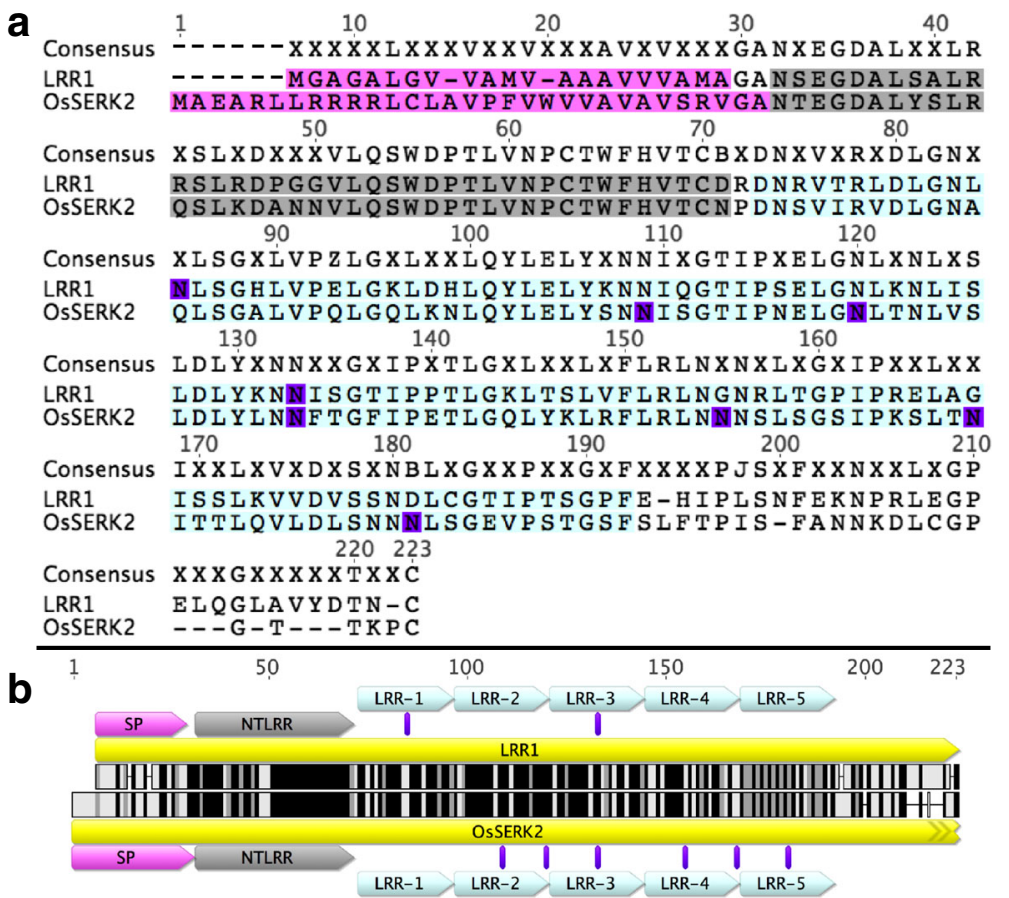

Fig. 1 LRR1 has sequence identity similar to the extracellular region OsSERK2. a Amino acid alignment of LRR1 (Os01g59440) and the consensus region of OsSERK2 (Os04g38480). Alignments were performed using Geneious R6.1.8 (Kearse et al. 2012). ' $X$ ' in the consensus sequence signifies a non-conserved amino acid. Dashes indicate gaps. b Alignment of LRR1 and OsSERK2 showing predicted structural motifs. SP: Secretion peptide (pink), NTLRR: N-terminal LRR capping domain (grey), LRR: Leucine rich repeat (blue), PG: Predicted glycosylation site (purple). Colors of the amino acid sequences indicate conservation. Black: conserved, grey: synonymous, white: nonsynonymous, dashes indicate gaps

$\left(\mathrm{T}_{1}\right)$ (Additional file 2: Figure S2). A fourth line, 13, which was not inoculated in the $\mathrm{T}_{0}$ generation, was also advanced to the $\mathrm{T}_{1}$ generation. Xoo inoculations were repeated in the $T_{1}$ generation and enhanced susceptibility was observed in lines 1 and 4 . Enhanced susceptibility was not statistically significant in transgenic plants of line 3, although the mean lesion length was longer (combined average of $5.6 \pm 2.4 \mathrm{~cm}$ ) compared to line $13(4.0 \pm 1.7 \mathrm{~cm})$ and XA21 $(4.1 \pm 1.3 \mathrm{~cm})$ (Additional file 3: Figure S3). In the $T_{2}$ generation, 6-weeks old rice from lines $1-1,1-5,3-1,3-8,4-1,4-3,13-1$, and 13-7 were inoculated with PXO99. Fourteen days after inoculation, XA21-LRR1Ri rice lines 1-1, 1-5, $3-1,3-8,4-1,4-3$ displayed enhanced susceptibility to PXO99 compared to Xa21, line 13-1, and 13-7. Kitaake plants were fully susceptible to PXO99 (Fig. 2b and Additional file 4: Figure S4). Silencing of LRR1 was confirmed in three independently transformed lines, 1-5-1, 3-8-6, and 4-1-2 (Fig. 2a), and three additional plants, 1-6-22, 3-2-22, and 4-3-25 (Additional file 5: Figure S5). However, line 13-6-1, which does not show the enhanced susceptibility phenotype did not have reduced expression of LRR1 (Fig. 2a). This result indicates that silencing of LRR1 impairs XA21-mediated immunity.

\section{XA21-LRR1Ri Lines Have Reduced Levels of Xa21 Transcripts}

The expression level of $\mathrm{Xa21}$ transcripts was assayed in XA21-LRR1Ri lines 1-5-1, 3-8-6, 4-1-2, and 13-6-1 (Fig. 3). XA21-LRR1Ri lines 1-5-1, 3-8-6, and 4-1-2, had significant reductions in $\mathrm{Xa21}$ transcript accumulation. However, XA21-LRR1Ri line 13-6-1 does not reduce expression of LRR1 and did not have reduction in Xa21 transcripts (Fig. 3). A pairwise alignment of LRR1Ri and XA21 was performed. The shared nucleotide identity across the consensus region was $56.8 \%$ with no consecutive stretch longer than 8 nucleotides, suggesting that XA21 silencing was not caused by off-target silencing of the LRR1Ri construct (Additional file 6: Figure S6). In contrast, the expression of Os11g36180, the Kitaake XA21 homolog, did not have statistically significant reduction in expression in any tested XA21-LRR1Ri line compared to XA21 (Additional file 7: Figure S7). Additionally, we tested if LRR1 silencing affected other immune receptors or components of XA21-mediated immunity. Transcript abundance was not reduced in two other immune receptors, rice CHITIN ELICITOR RECEPTOR KINASE 1 (OsCERK1) and OsFLS2 (Additional file 7: Figure S7). Similarly, reduced expression was not observed in the XA21 signaling components OsSERK2, 


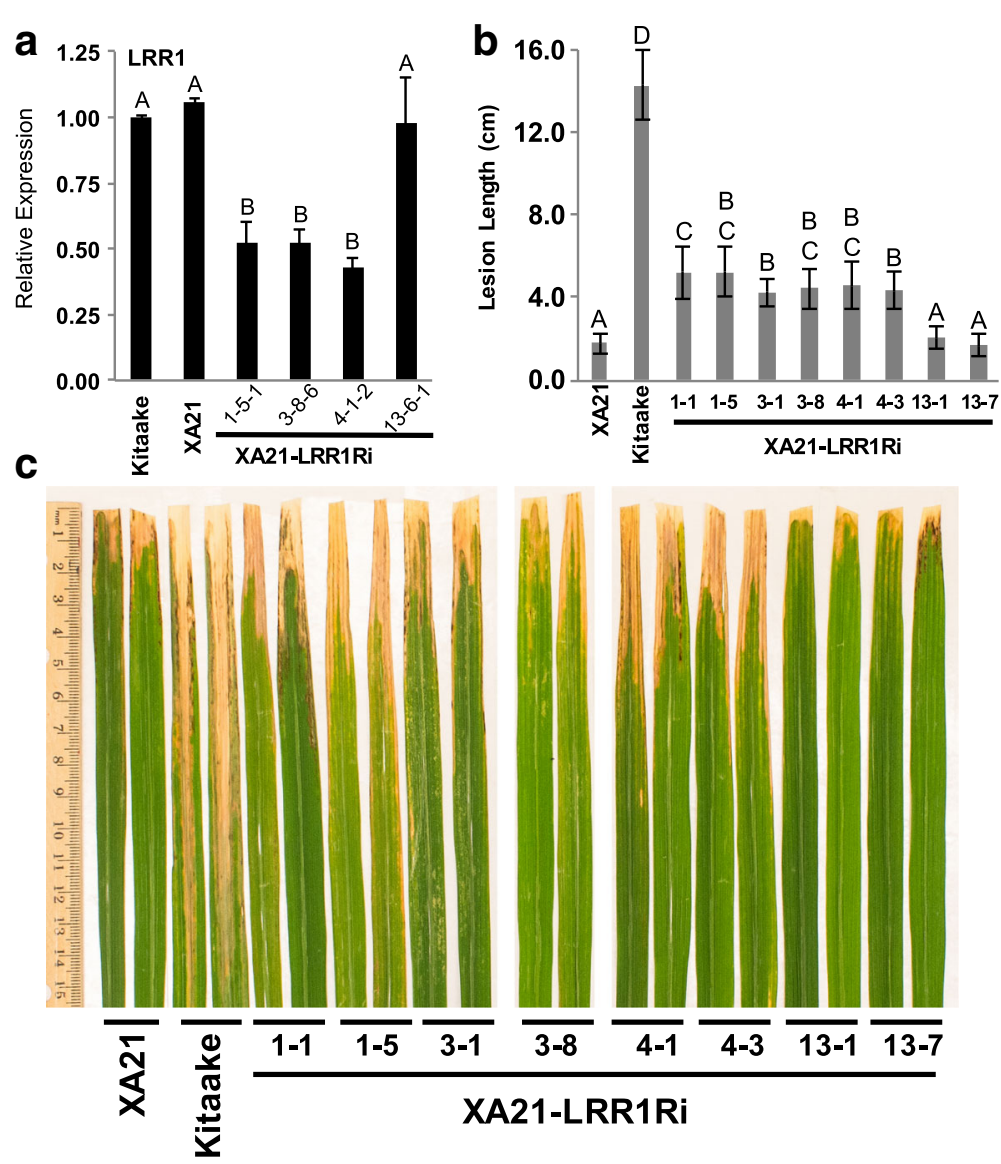

Fig. 2 Silencing of $L R R 1$ in the XA21 genetic background enhances susceptibility to Xoo. a Relative expression level of LRR1 in four independent transgenic rice lines. Bars depict the average and standard deviation of $L R R 1$ expression normalized to Kitaake of two technical replicates.

Different letters indicate a significant difference in gene expression $(P<0.05$, ANOVA, Tukey-HSD). This experiment was repeated three times with similar results. $\mathbf{b}$ Lesion length of $\mathrm{T}_{2}$ generation XA21-LRR1Ri plants 14 days after inoculation with PXO99. Bars indicate the average lesion length and standard deviation of ten to 15 combined plants that had one to six inoculated leaves each. Different letters indicate a significant difference in lesion length ( $P<0.05$, Kruskal-Wallis test, Dunn's post-hoc test with Benjamini-Hochberg correction). Lesion length of individual $T_{2}$ plants from this experiment are shown in Additional file 4: Figure S4. Experimental results were repeated three times, with similar results. c Two representative inoculated leaves from each line were photographed at the time of lesion scoring

$\mathrm{XB} 3, \mathrm{XB15}, \mathrm{XB} 21$, or XB24, indicating that $L R R 1 R i$ specifically reduced $\mathrm{Xa} 21$ transcript levels. To determine if silencing of OsSERK2 also reduces Xa21 transcript levels, we compared $\mathrm{Xa} 21$ expression between XA21 rice and a homozygous line expressing an OsSERK2 silencing construct (XA21-OsSERKRi) (Chen et al. 2014). No statistical difference in Xa21 expression was detected, indicating that silencing of OSSERK2 does not significantly reduce Xa21 transcript levels (Additional file 8: Figure S8).

To assess if $\mathrm{Xa21}$ transcript reduction was specific to the LRR1Ri construct, two sibling LRR1 silenced lines from line 3 that had lesion lengths measured in the $T_{0}$ and $\mathrm{T}_{1}$ generation were analyzed for $X a 21$ expression in the $T_{2}$ generation. XA21-LRR1Ri line 3-2 is homozygous for the transgene. Sibling line, XA21-LRR1Ri 3-3, is a null-segregant lacking the LRR1Ri transgene. Reduction in $\mathrm{Xa21}$ transcripts was specific to line 3-2, which contains the LRR1Ri construct, while line 3-3 did not display similarly reduced levels of $\mathrm{Xa21}$ transcripts (Additional file 9: Figure S9). Taken together, these results indicate that $\mathrm{Xa21}$ transcript reduction correlates with the LRR1 silencing and is not due to aberrant expression of $\mathrm{Xa21}$ in the genetic background used for transformation.

\section{XA21-LRR1Ri Plants Fail to Express XA21 Genetic Markers After RaxX21-sY Peptide Treatment}

We previously demonstrated that treatment of XA21 leaves with sulfated RaxX21-sY peptides induces expression of specific genetic markers. In contrast, treatment of XA21 plants with non-sulfated RaxX21 peptides do not induce these marker genes (Pruitt et al. 2015). To determine if the XA21-LRR1Ri plants fail to respond to RaxX, rice leaves from $L R R 1$ silencing lines were treated 


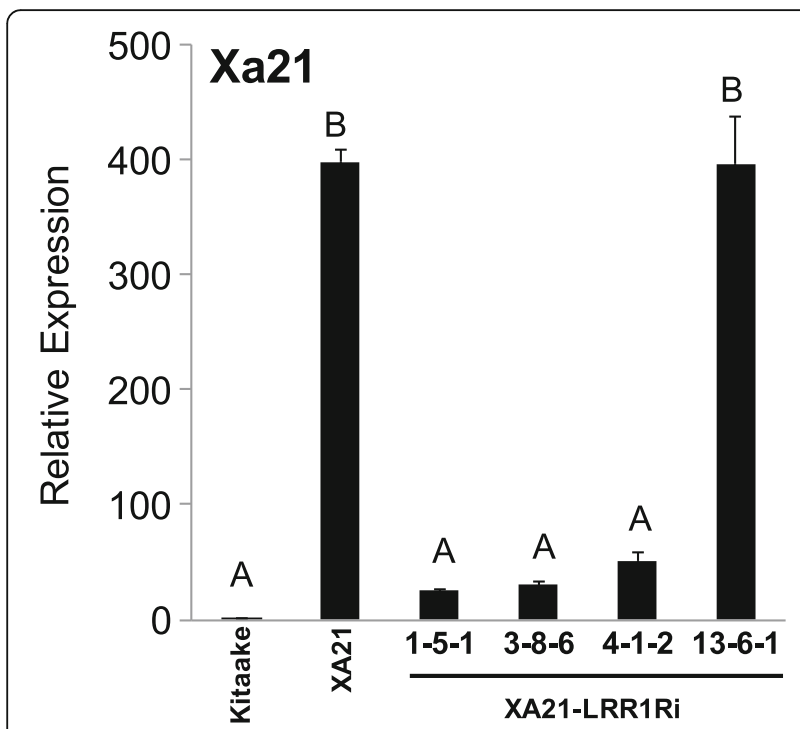

Fig. 3 Xa21 expression is reduced in XA21-LRR1Ri rice lines. Relative expression of $\mathrm{X} a 21$ in four independent transgenic rice lines. Bars depict the average and standard deviation of Xa21 expression normalized to Kitaake of two technical replicates. Different letters indicate a significant difference in gene expression ( $P<0.05$, ANOVA, Tukey-HSD). This experiment was repeated three times with similar results

with RaxX21-sY peptides. Six hours after RaxX21-sY peptide application, the expression level of $P R 10 b$ (Fig. 4a), Os10g28299 (Fig. 4b), or Os04g10010 (Fig. 4c) were assayed in Kitaake, XA21, and XA21-LRR1Ri lines. We observed that RaxX21-sY treatment of XA21 plants induced expression of these marker genes as previously reported (Pruitt et al. 2015). In contrast, RaxX21-sY peptide treatment failed to induce the expression of the three stress-related genes in the XA21-LRR1Ri lines. These results suggest that a reduced ability to initiate an XA21-mediated immune response contributes to the enhanced susceptibility observed in XA21-LRR1Ri plants.

\section{Overexpression of $L R R 1$ in the Kitaake Background Does not Confer Resistance to Xoo}

Previously it was reported that overexpression of $L R R 1$ did not enhance rice resistance to Xoo strain LN44 in the Japonica rice variety SN1033 (Zhou et al. 2009). Because we observed that LRR1 expression is required for XA21-mediated immunity, we examined if overexpression of LRR1 is sufficient to enhance resistance to PXO99 in the rice variety Kitaake, which lacks Xa21. The overexpression of LRR1 in the XA21 background was not tested because XA21 plants are already resistant to $X o o$. We generated sixteen transgenic rice plants expressing LRR1 under the control of the maize ubiquitin promoter (LRR1ox) by Agrobacterium-mediated plant transformation. Fourteen of 16 transgenic lines contained the transgene as determined by PCR analysis.
Kitaake, XA21, and ten $\mathrm{T}_{0}$ lines were inoculated with PXO99. Four of ten lines displayed significantly shorter lesion lengths than Kitaake (Additional file 10: Figure S10). Two additional $\mathrm{T}_{0}$ lines, LRR1ox-15 and LRR1ox16, were inoculated at a later date and also displayed shorter lesions than Kitaake (Additional file 10: Figure S10). Four $\mathrm{T}_{0}$ Lines, LRR1ox- $7,-10,-12$, and -16 were advanced to the next generation. Inoculations were repeated in the $T_{1}$ generation. LRR1ox lines $7,10,12$, and 16 were all fully susceptible to Xoo (Additional file 11: Figure S11).

Overexpression of LRR1 in LRR1ox lines 7-10, 7-12, $10-2,10-3,12-1,12-3,16-1$ and $16-2$ was confirmed in the $\mathrm{T}_{2}$ generation by quantitative RT-PCR (Fig. $5 \mathrm{a}$ ) and the plants were inoculated with PXO99. Lesion development was measured 14 days after inoculation and no significant difference in lesion development was found compared with the nontransgenic Kitaake control plants (Fig. 5b). The partial resistance phenotype of LRR1ox lines in the $\mathrm{T}_{0}$ generation was not observed in the $T_{1}$ or $T_{2}$ generation inoculations. Because $T_{0}$ plants are regenerated from rice calli, we hypothesize that the shorter lesions are due to differences in the leaf developmental stage at the time of inoculation compared to Kitaake, which was germinated from seed. Together, these results indicate that overexpression of $L R R 1$ in Kitaake is not sufficient to enhance resistance to Xoo, thus confirming the previous report by Zhou et al. 2009, but in a different genetic background.

\section{Discussion}

To elucidate the function of XA21 and the XA21 binding protein XB21, we characterized LRR1, which interacts with XB21. LRR1 is a leucine rich repeat containing protein with similarity to the LRR domain of OsSERK2 (Chen et al. 2014) (Fig. 1), which is a co-receptor for XA21. Silencing of LRR1 in the resistant XA21 genetic background conferred enhanced susceptibility to Xoo and reduced the expression of $\mathrm{Xa21}$ (Fig. 2b). These results suggest that LRR1 is required for $\mathrm{Xa21}$ transcription.

\section{LRR1 Functions in Plant Immunity}

Our results support previous studies demonstrating a role for LRR1 in plant immunity. For example, overexpression of rice LRR1 in Arabidopsis thaliana and Chinese cabbage is sufficient to confer enhanced resistance against the bacterial pathogens Pseudomonas syringae pv. tomato DC3000 (Pst DC3000) (Zhou et al. 2009; Zhou et al. 2010) and Pectobacterium carotovorum subsp. carotovorum (Park et al. 2012) respectively. In contrast to these results, we found overexpression of $L R R 1$ in the Kitaake rice variety did not enhance resistance to Xoo (Fig. 5b). A similar result was reported by Zhou et al. 2009, who found that overexpression of $L R R 1$ did not enhance resistance to Xoo 

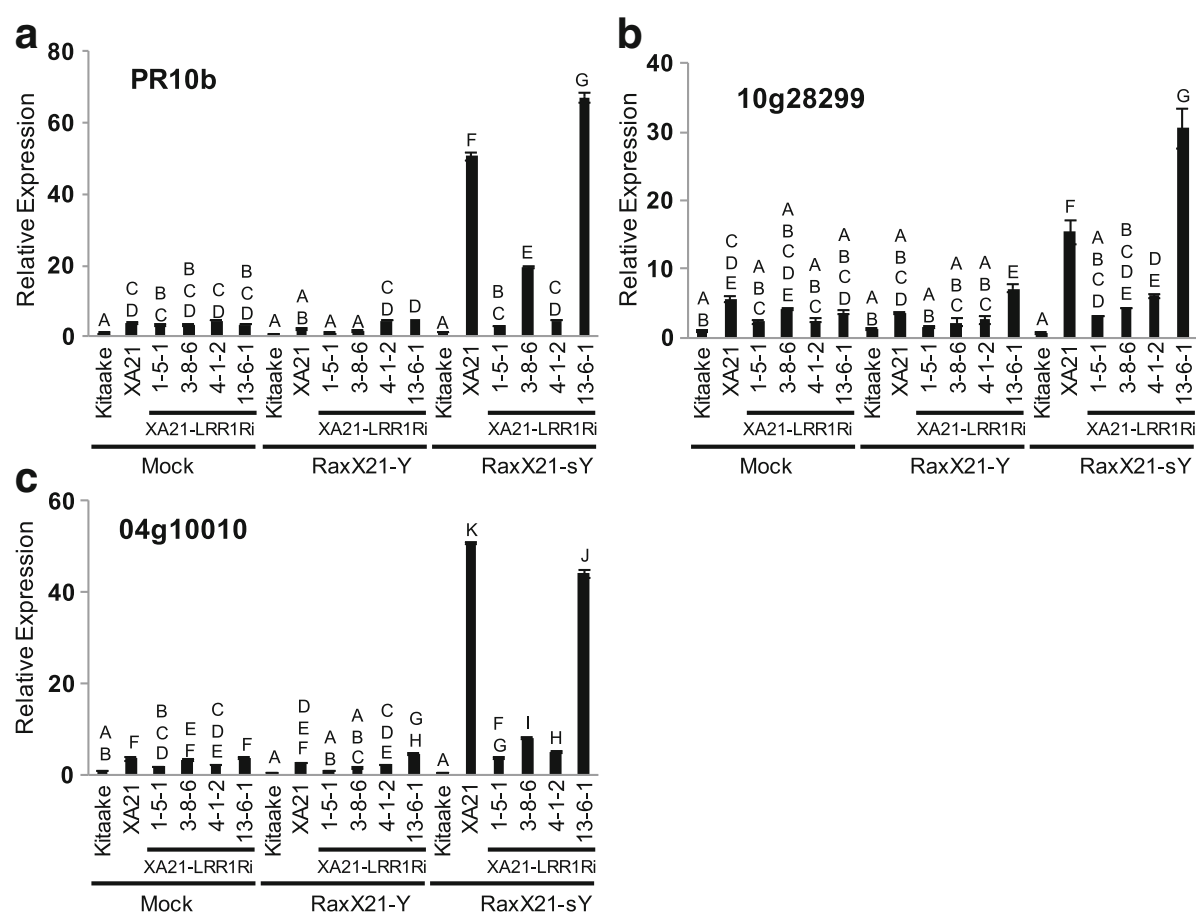

Fig. 4 XA21-LRR1Ri rice plants are unresponsive to RaxX21-sY peptide treatment. Gene expression of PR10b (a), $10 \mathrm{~g} 28299$ (b), and $04 \mathrm{~g} 10010$ (c) $6 \mathrm{~h}$ after 500nM application of RaxX21, RaxX21-sY, or mock treatment in mature leaves of the four independent XA21-LRR1Ri rice lines. Bars depict the average and standard deviation of expression level normalized to Kitaake mock treatment of two technical replicates. Different letters indicate a significant difference in gene expression $(P<0.05$, ANOVA, Tukey-HSD). Experiment was performed two times with similar results

strain LN44 in the rice variety SN1033 (Zhou et al. 2009). The LRR1 ortholog of pepper, CaLRR1, which is induced upon infection with Xanthomonas campestris pv. vesicatoria (Xcv) (Jung et al. 2004), did not enhance resistance to Pst DC3000 when overexpressed in Arabidopsis (Choi et al. 2012). However, overexpression enhanced callose deposition and reduced conidiospore production during Hyaloperonospora arabidopsidis infection (Choi et al. 2012). CaLRR1 silenced pepper plants have enhanced susceptibility to $\mathrm{Xcv}$ and reduced $\mathrm{H}_{2} \mathrm{O}_{2}$ production (Hwang et al. 2014). They also have reduced production of defense gene expression and total SA accumulation (Choi et al. 2012). Together, our study and previous reports demonstrate that the LRR1 family of proteins have diverse effects on plant immune responses.

\section{The Function of LRR1 is Distinct from OsSERK2}

OsSERK2 is required for OsBRI1-mediated development and regulating XA21, XA3 and OsFLS2-mediated immune responses (Chen et al. 2014). XA21 rice plants with reduced levels of OsSERK2 (XA21-OsSERK2Ri) have compromised resistance to Xoo (Chen et al. 2014). Despite the structural similarity between the LRR domains of LRR1 and the OsSERK2, it is likely that their functions are distinct. The intracellular domain of OsSERK2 is essential for the interaction between OsSERK2 and the intracellular domains of XA21, XA3, OsFLS2, and OsBRI1 (Chen et al. 2014). Direct interaction with these cellsurface receptors is thought to be critical for OsSERK2 functionality. Unlike OsSERK2, LRR1 lacks an intracellular domain. When XA21-LRR1Ri rice lines expressed $L R R 1 R i$, we consistently detected a significant reduction in Xa21 transcript abundance (Fig. 3). Because LRR1Ri causes reduced $\mathrm{Xa} 21$ expression, we determined whether silencing OsSERK2 had a similar effect. However, XA21OsSERK2Ri rice plants do not have reduced Xa21 transcript levels (Additional file 8: Figure S8). This suggests that the enhanced susceptibility conferred by LRR1Ri is mechanistically different than OsSERK2 silencing in rice plants.

\section{LRR1 Interacts With OsHIR1}

The results of our study suggest a new role of LRR1 in regulating the expression of a rice immune receptor. Previous studies demonstrated that LRR1 interacts with HYPERSENSITIVE INDUCED REACTION PROTEIN 1 (OsHIR1) in rice (Zhou et al. 2010; Zhou et al. 2009). While the role of OsHIR1 in rice immunity has not been extensively studied, proteomic and gene expression analysis of XA21 rice plants indicate that OsHIR1 expression increased after inoculation with Xoo. OsHIR1 is also thought to be post-translationally modified in XA21 

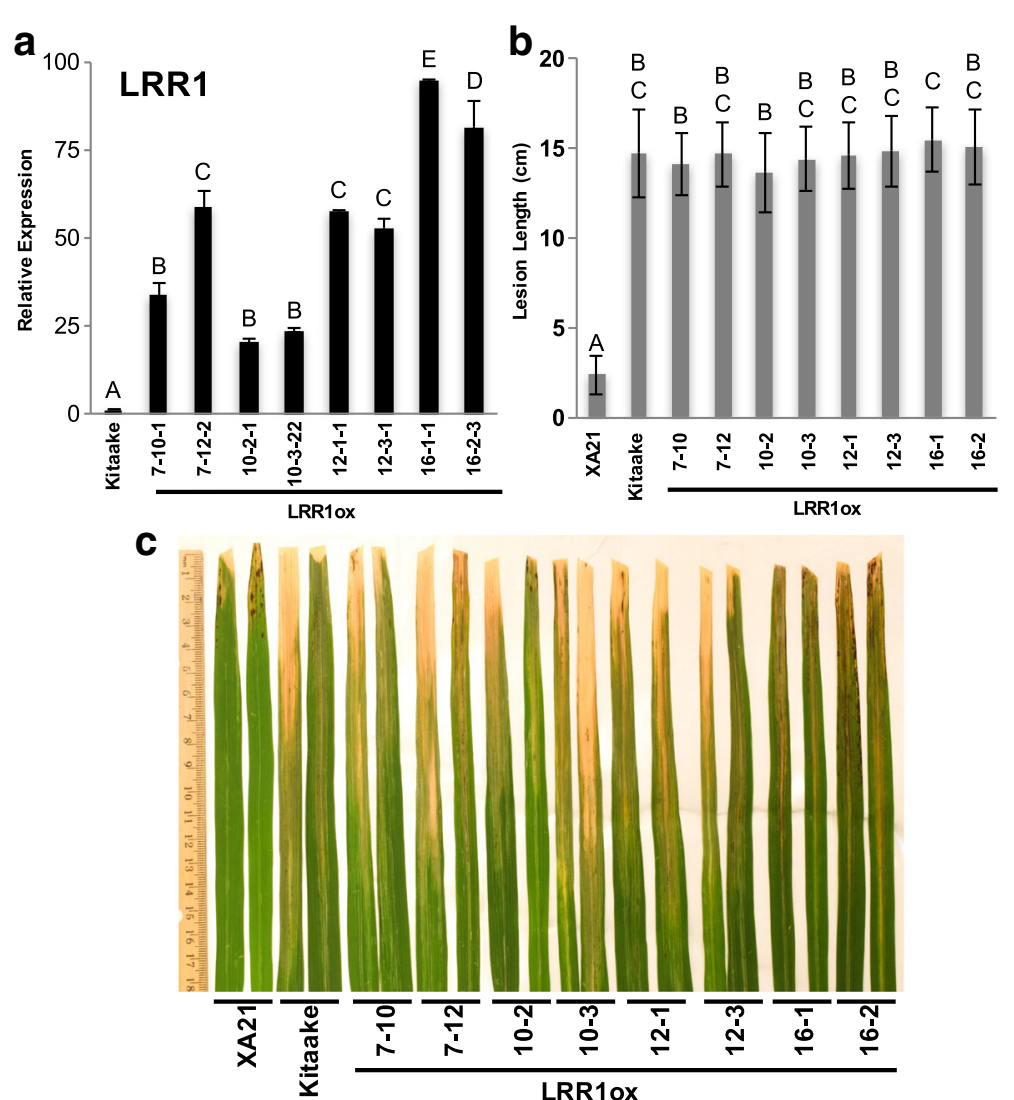

Fig. 5 Kitaake rice overexpressing $L R R 1$ are susceptible to Xoo. a Bars depict the average and standard deviation of $L R R 1$ expression normalized to Kitaake of two technical replicates. Different letters indicate a significant difference in gene expression $(P<0.05$, ANOVA, Tukey-HSD). This experiment was repeated three times with similar outcomes. b Lesion length of LRR1ox 14 days after inoculation with PXO99. Bars indicate the mean lesion length and standard deviation on four to nine plants that had two to nine inoculated leaves. Different letters indicate a significant difference in lesion length $(P<0.05$, Kruskal-Wallis test, Dunn's post-hoc test with Benjamini-Hochberg correction). This experiment was repeated 4 times with similar results. c Two representative inoculated leaves from each line were photographed th the time of lesion scoring

plants challenged with Xoo (Chen et al. 2007), suggesting that OsHIR1 might function in XA21mediated immunity.

The orthologous proteins CaLRR1 and CaHIR1 also interact in pepper (Jung and Hwang 2007). Overexpression of OsHIR1 (Zhou et al. 2010) or CaHIR1 (Jung and Hwang 2007) in Arabidopsis both promoted the formation of cell death lesions (Jung and Hwang 2007). The cell death symptom of CaHIR1 overexpressing plants was alleviated by simultaneously overexpressing CaLRR1 (Jung and Hwang 2007). CaHIR1 positively regulates RESISTANCE TO PSEUDOMONAS SYRINGAE PROTEIN 2 (RPS2)-mediated immunity to $X c v$ (Choi et al. 2013; Jung and Hwang 2007; Jung et al. 2004; Choi et al. 2011). During infection by $X c v$, the virulence factor FILAMENTOUS HAEMAGGLUTININ ADHESIN 1 (Fha1) targets CaHIR1 where it modulates cell death and suppresses pepper PR gene expression (Choi et al. 2013). Under normal conditions, CaLRR1 and CaHIR1 both co-localize at the plasma membrane (Choi et al. 2011), while CaHIR1-Fha1 heterodimers were mostly found in endosomes during Xcv infection (Choi et al. 2013). These results suggest that the plasma membrane localization of CaHIR1 is important for the CaHIR1mediated resistance.

\section{LRR1 is Required for XA21 Expression}

The transcriptional regulation of plant immune receptors is not well studied, and we do not know how LRR1 expression is linked to the transcription of $\mathrm{Xa21}$. A recent report identified four receptor-like cytoplasmic kinases that also modulate proA-Xa21 expression in the Kitaake genetic background (Zhou et al. 2016). Future experiments are needed to understand whether LRR1 is regulating Xa21 through the same pathway or parallel pathways.

While we did not observe high complementarity between Xa21 and LRR1 (Additional file 6: Figure S6), we cannot rule out the possibility that the primary siRNA derived from processing of $L R R 1 R i$ or a transitive secondary 
siRNA produced upon cleavage of $L R R 1$ have sufficient complementarily with $\mathrm{Xa21}$ to trigger mRNA cleavage. However, it is known that the expression of plant immune receptors must be tightly regulated for optimal growth and overexpression of plant immune receptors can decrease plant fitness (Bergelson and Purrington 1996; Huot et al. 2014). For example, a $9 \%$ reduction in fitness was observed in Arabidopsis ecotype Bla-2 after the resistance gene RESISTANCE TO P. SYRINGAE PV MACULICOLA 1 (RPM1) was inserted (Tian et al. 2003).

Previous studies have reported that proteins involved in membrane trafficking including clatharin adapters, accessory proteins, and endosomal proteins facilitate the modification of gene expression (Pyrzynska et al. 2009). In rice, OsHIR1 and LRR1 are detected in endosomes and the plasma membrane (Zhou et al. 2009). LRR1 enhances the plasma membrane localization of OsHIR1 (Zhou et al. 2010). It is possible that XB21, which interacts with LRR1 in yeast, facilitates the transport of proteins such as OsHIR1 from the plasma membrane to endosomes. XB21 contains a C-terminal J domain. This class of protein has been associated with the uncoating of clatharin-coated vesicles, which occurs after endocytic vesicles bud off from the plasma membrane (Lemmon 2001; Kampinga and Craig 2010). These studies suggest that endosomal accumulation of OsHIR1 and other plasma membrane proteins may influence the expression of genes encoding receptors such as XA21.

\section{Conclusions}

Rice LRR1 and its pepper ortholog were previously reported to enhance resistance to bacterial pathogens in Arabidopsis and Chinese cabbage. In this study, we identified a new role of LRR1: transcriptional regulation of the rice immune receptor $\mathrm{Xa21}$.

\section{Methods}

\section{Plant Material and Growing Conditions}

Rice plants (Oryza sativa L.) were germinated in water filled petri dishes at $28{ }^{\circ} \mathrm{C}$ and transferred to the greenhouse $\left(\sim 28-30{ }^{\circ} \mathrm{C}, 75-85 \%\right.$ humidity) after 7 days. Rice plants were grown in 80/20 (sand/peat) soil mixture. Greenhouse grown rice plants were supplemented with artificial light from November to April to obtain $14 \mathrm{~h}$ days. Six-week old greenhouse grown plants were transferred to growth chambers for inoculation. Growth chambers for inoculation were set at $28{ }^{\circ} \mathrm{C}$ Day/26 ${ }^{\circ} \mathrm{C}$ Night, $85 \%$ humidity, $16 \mathrm{~h}$ days. Inoculations were performed a minimum of 3 days after transferring plants to reduce plant stress from transfer. For gene expression assays, rice plants were transferred to growth chambers $\left(22{ }^{\circ} \mathrm{C}, 80 \%\right.$ humidity, $16 \mathrm{~h}$ days) after 7 days and grown in hydroponics conditions using Hoagland solution changed twice weekly. Fully expanded leaves from 4- week old hydroponics grown plants were cut into $1.5 \mathrm{~cm}$ strips and floated overnight in Milli-Q $\mathrm{H}_{2} \mathrm{O}$ to reduce wounding. Mock, RaxX21-Y or RaxX21-sY peptide treatments were applied at a final concentration of $500 \mathrm{nM}$ for $6 \mathrm{~h}$. Leaf fragments were flash frozen in liquid nitrogen and stored at $-80{ }^{\circ} \mathrm{C}$ for further analysis.

\section{Xoo Inoculations}

Philippines race 6 strain of Xoo (PXO99Az) was plated on peptone sucrose agar plates for 3 days then suspended in Milli-Q $\mathrm{H}_{2} \mathrm{O}$ to a concentration of $\mathrm{OD}_{600}$ $=0.5$. Fully expanded and healthy rice leaves were inoculated approximately $2 \mathrm{~cm}$ from the leaf tip using the scissors- clip method (Chern et al. 2005). Lesion lengths were measured 12 or 14 days after inoculation.

\section{Plasmid Construction}

Rice cDNA encoding full-length LRR1 (Os01g59440) was amplified from rice cDNA using the primers $5^{\prime}$ - CACCATGGGGGCGGGGGCGCTGGG -3' (LRP-FL-S) and 5' - CTAGCAGTTGGTGTCATATACAGC-3' (LRP-FL-A S). PCR products were cloned into pENTR/D-TOPO vector (Invitrogen) using the instructions provided by the manufacturer. The proper sequence was confirmed by sequencing. The LRR1 overexpression construct was constructed by recombining the LRR $1 / \mathrm{pENTR} / \mathrm{D}-\mathrm{TOPO}$ construct into the gateway-compatible Ubi-CAMBIA1300 (Chern et al. 2005). For E. coli expression, XB21/ pENTR/D-TOPO, which encodes full-length XB21 protein (Os12g36180), was amplified from rice cDNA using primers 5'-CACCATGGACGACTTCCAGGGCCTCCT GGCC-3' and 5' $^{\prime}$-TTAGAAGAGTTCCTCTGAGTTGA ATTTG-3' (Park et al. manuscript in preparation). LRR1/ pENTR/D-TOPO and XB21/pENTR/D-TOPO were recombined into pDEST15 and pDEST17 expression vectors respectively. The pGEX-1 vector (Chen et al. 2010) was used for expression of GST. For silencing of $L R R 1$, the 357 bp fragment shared by three predicted isoforms of LRR1 was amplified from rice cDNA using the primers $5^{\prime}$-CACCTTGGGAATTTGAACTTATCT GGTC-3' (LRP-RNAi-S) and 5'-AGTTGCTTAGGGG AATGTGCTC-3' (LRP-RNAi-AS), and cloned into $\mathrm{pENTR/D-TOPO}$ vector to produce LRR1Ri/pENTR/DTOPO. The LRR1Ri/pENTR/D-TOPO was recombined into the pANDA silencing vector (Miki and Shimamoto 2004) using gateway LR clonase (Invitrogen).

\section{Rice Transformation}

Kitaake rice plants and homozygous Kitaake plants carrying Xa21 with its native promoter, XA21 23A-1-14 (referred to as XA21 in this paper) (Park et al. 2010), were transformed as described previously (Chern et al. 2005) using Agrobacterium strain EHA105 to infect rice 
calli. Transformants carrying the transgenes were selected using hygromycin and later confirmed by PCR.

\section{Yeast two-Hybrid}

The N-terminal deleted XB21 (XB21 $\triangle \mathrm{NT})(\mathrm{Os} 12 \mathrm{~g} 36180)$ covering amino acids 433-926 was amplified from rice cDNA using the primers 5 -CACCATGTCAATAGATG AACTGGAAGATTTT-3' (XB21-A-S) and 5'-TTAGA AGAGTTCCTCTGAGTTGAATTTG-3' (XB21-FL-AS), cloned into the yeast two-hybrid bait vector pMC86, and transformed into yeast strain HF7c MATa. Transformation of target yeast (Y187) used cDNA obtained from a Hybrizap (Stratagene) yeast two-hybrid library derived from leaf MRNA of 7-week-old Indica rice cultivar IRBB21. Screening was performed as described previously (Seo et al. 2011).

\section{Gene Expression Assays and Quantitative RT-PCR}

RNA was extracted from rice plants using Spectrum Plant Total RNA Kit (Sigma) followed by DNase treatment performed using Turbo DNA-free DNase (Ambion). RNA integrity was confirmed by agarose gel electrophoresis (with $0.1 \% \mathrm{SDS}$ ). $2 \mu \mathrm{g}$ of RNA was used as template for cDNA synthesis, which was performed using the High-Capacity cDNA Reverse Transcription Kit (Applied Biosystems). Quantitative RT-PCR using SsoFast EvaGreen Supermix (Bio-Rad) was performed on a CFX96 Real-Time system coupled to a C1000 Thermal Cycler (Bio-Rad) with the conditions: $95{ }^{\circ} \mathrm{C}$ for $10 \mathrm{~s}, 60^{\circ} \mathrm{C}$ for $10 \mathrm{~s}, 40$ cycles. Amplicon specificity was confirmed by checking the melting curve at the completion of 40 cycles. Expression of actin was used to normalize samples for analysis. Technical replication was performed by amplifying the same cDNA sample 2 or 3 times as described in the individual figure legends.

\section{Statistical Analyses}

The normal distribution of data was tested using the Shapiro-Wilk test. Normally distributed data was tested using a one-way analysis of variance (ANOVA) test. Statistically significant results were followed up with a Tukey's honest significance (Tukey-HSD) post-hoc test using JMP v.13.1 software (SAS Institute Inc.). A Kruskal-Wallis nonparametric test was applied to data that violated normality assumptions. Statistically significant results were followed up with a Dunn post-hoc test with Benjamini-Hochberg correction (Ogle 2017) using R v.3.3.2 (Team 2016). Significance for all statistical tests was predetermined at $p<0.05$.

\section{Primers}

A complete list of primers used in this study can be found in Additional file 12: Table S1.

\section{Additional files}

Additional file 1: Figure S1. His-XB21 and GST-LRR1 interact in vitro. His-XB21, GST, and GST-LRR1 proteins were immunoprecipitated from E. coli using Ni-NTA resin and Glutathione Sepharose 4B respectively (lanes 1,2,3). His-XB21 was added to bound GST and GST-LRR1. After co-incubation and centrifugation, unbound supernatant (lanes 4,5) and co-immunoprecipitated (Co-IP) (lanes 5,6) fractions were obtained. The samples were then subjected to SDS-PAGE for western analysis using anti-HIS and anti-GST antibodies. This experiment was performed twice with similar results. (PDF $328 \mathrm{~kb}$ )

Additional file 2: Figure S2. $T_{0}$ generation $X_{00}$ inoculation of XA21LRR1Ri lines. Lesion length of XA21-LRR1Ri plants 12 days after inoculation with PXO99. Bars indicate the average lesion length and standard deviation on individual rice plants that had three to 19 inoculated leaves. Different letters indicate a significant difference in lesion length $(P<0.05$, Kruskal-Wallis test, Dunn's post-hoc test with Benjamini-Hochberg correction). (PDF $13 \mathrm{~kb}$ )

Additional file 3: Figure S3. $T_{1}$ generation $X_{00}$ inoculation of XA21LRR1Ri lines. Lesion length of XA21-LRR1Ri plants 12 days after inoculation with PXO99. Bars indicate the average lesion length and standard deviation on individual rice plants that had one to four inoculated leaves. Different letters indicate a significant difference in lesion length $(P<0.05$, Kruskal-Wallis test, Dunn's post-hoc test with Benjamini-Hochberg correction). Gray bars indicate the presence of the LRR1Ri construct, white bars indicate null-segregants. (PDF $17 \mathrm{~kb}$ )

Additional file 4: Figure S4. $T_{2}$ generation $X_{00}$ inoculation of XA21LRR1Ri lines. Lesion length of XA21-LRR1Ri plants 14 days after inoculation with PXO99. Bars indicate the average lesion length and standard deviation on individual rice plants that had one to six inoculated leaves. Gray bars indicate the presence of the LRR1Ri construct, white bars indicate null-segregants. Different letters indicate a significant difference in gene expression ( $P<0.05$, ANOVA, Tukey-HSD). (PDF $28 \mathrm{~kb}$ )

Additional file 5: Figure S5. $\angle R R 1$ expression is reduced in XA21-LRR1R rice lines. Relative expression of $\angle R R 1$ in three independent transgenic rice lines. Bars depict the average and standard deviation of $L R R 1$ expression normalized to XA21 of two technical replicates. (PDF $15 \mathrm{~kb}$ )

Additional file 6: Figure S6. Pairwise alignment of LRR1Ri and XA21. The region of LRR1 used to develop the LRR1Ri vector was aligned to XA21 using Geneious R6.1.8 (Kearse et al. 2012). Nucleotide numbers refer to the original sequences. Highlighted in black are the conserved nucleotides between the two sequences. The shared identity within the consensus region is $56.8 \%$. (PDF $40 \mathrm{~kb}$ )

Additional file 7: Figure S7. $\angle R R 1$ silencing does not reduce the expression of three rice receptor kinases or components of XA21mediated immunity. Relative expression of OsSERK2, Os11936180, OsFLS2, OsCERK1, XB3, XB15, XB21, and XB24 in four independent XA21-LRR1Ri transgenic rice lines. Bars depict the average and standard deviation of expression level normalized to XA21 of three technical replicates. Different letters indicate a significant difference in gene expression $(P<0.05, A N O V A$, Tukey-HSD). This experiment was repeated at least two times with similar results. (PDF $21 \mathrm{~kb}$ )

Additional file 8: Figure S8. XA21-OsSERK2Ri plants do not reduce Xa21 expression. Relative expression of OsSERK2 (A) and Xa21 (B) in Kitaake, XA21, and XA21-OsSERK2Ri (homozygous line A814) transgenic rice. Bars depict the average and standard deviation of expression level normalized to XA21 of two technical replicates. Different letters indicate a significant difference in gene expression $(P<0.05$, ANOVA, Tukey-HSD). This experiment was repeated three times with similar results. (PDF $23 \mathrm{~kb}$ )

Additional file 9: Figure S9. XA21-LRR1Ri null segregants do not have reduced levels of Xa21 expression. Xa21 expression of six individual plants per line. Line 3-2 is homozygous for the LRRTRi transgene. Line 3-3 is a null segregant for $L R R 1 R i$. Bars depict the average and standard deviation of Xa21 expression normalized to Kitaake of two technical replicates. Different letters indicate a significant difference in gene expression $(P<0.05$, ANOVA, Tukey-HSD). (PDF $31 \mathrm{~kb}$ )

Additional file 10: Figure S10. $T_{0}$ generation $X_{00}$ inoculation of LRR1ox lines. Lesion length of LRR1ox plants 14 days after inoculation 
with PXO99. Bars indicate the average lesion length and standard deviation on individual rice plants that had four to 13 inoculated leaves. Different letters indicate a significant difference in lesion length $(P<0.05$, Kruskal-Wallis test, Dunn's post-hoc test with Benjamini-Hochberg correction). (PDF $11 \mathrm{~kb}$ )

Additional file 11: Figure S11. $T_{1}$ generation $X_{00}$ inoculation of LRR1ox lines. Lesion length of LRR1ox plants 14 days after inoculation with PXO99. Bars indicate the average lesion length and standard deviation on individual rice plants that had one to eight inoculated leaves. Different letters indicate a significant difference in lesion length $(P<0.05$, Kruskal-Wallis test, Dunn's post-hoc test with Benjamini-Hochberg correction). Gray bars indicate the presence of the LRR1ox construct, white bars indicate null-segregants. (PDF $24 \mathrm{~kb}$ )

Additional file 12: Table S1. Full list of primers used in this study. (XLSX $9 \mathrm{~kb})$

\section{Abbreviations}

ANOVA: Analysis of variance test; BAK1: BRI1-ASSOCIATED RECEPTOR KINASE 1; BRI1: BRASSINOSTEROID-INSENSITIVE 1; CERK1: CHITIN ELICITOR RECEPTOR KINASE 1; EFR: EF-TU RECEPTOR; elf18: 18 amino acid epitope of elongation factor-Tu; Fha1: FILAMENTOUS HAEMAGGLUTININ ADHESIN 1; flg22: 22 amino acid epitope of bacterial flagellin; FLS2: FLAGELLIN SENSING 2; HIR1: HYPERSENSITIVE INDUCED REACTION PROTEIN 1; LRR1: LEUCINE-RICH REPEAT PROTEIN 1; LRR1OX: LRR1 overexpression construct driven by the maize ubiquitin promoter; LRR1Ri: LRR1 RNAi silencing construct; LRRRLK: leucine-rich repeat receptor-like kinase; Pst DC3000: Pseudomonas syringae pv. tomato DC3000; PX099: Philippine Xoo strain PXO99AZ RaxX: REQUIRED FOR ACTIVATION OF XA21-MEDIATED IMMUNITY X; RaxX21-sY: 21 amino acid tyrosine sulfated epitope of RaxX; RPM1: RESISTANCE TO P. SYRINGAE PV MACULICOLA 1; RPS2: RESISTANCE TO PSEUDOMONAS SYRINGAE PROTEIN 2; SERK: SOMATIC EMBRYOGENESIS RECEPTOR KINASE; Tukey-HSD: Tukey's honest significance test; XA21: XOO RESISTANCE 21; XA21-LRR1Ri: XA21 rice plants containing the LRR1Ri construct; XB21: XA21 BINDING PROTEIN 21; XB21IPs: XB21 interacting proteins; XB21 $\triangle \mathrm{NT}$ : N-terminal deleted XB21; XCV: Xanthomonas campestris pv. vesicatoria; Xoo: Xanthomonas oryzae pv. oryzae

\section{Acknowledgements}

We thank Xia Xu for her contribution to the yeast two-hybrid screen.

\section{Funding}

This work was supported by the National Institute of Health (NIH, GM55962) and the National Science Foundation (NSF, IOS-0817738). DC was supported by the Monsanto Beachell-Borlaug International Scholars Program.

\section{Availability of data and materials}

Not applicable.

\section{Authors' contributions}

$P C$ generated the transgenic rice plants. DC, CP, NT performed the experiments. DC, CP, PR wrote the manuscript. All authors discussed the research and approved the manuscript.

\section{Competing interests}

The authors declare that they have no competing interests.

\section{Consent for publication}

Not applicable.

Ethics approval and consent to participate Not applicable.

\section{Publisher's Note}

Springer Nature remains neutral with regard to jurisdictional claims in published maps and institutional affiliations.

\section{Author details}

${ }^{1}$ Department of Plant Pathology and the Genome Center, University of California, Davis, Davis, CA 95616, USA. ${ }^{2}$ Present Address: Department of
Bioresources Engineering and PERI, Sejong University, Seoul 05006, Republic of Korea. ${ }^{3}$ Present Address: Department of Plant Pathology and Microbiology, University of California, Riverside, Riverside, CA 92521, USA.

Received: 16 February 2017 Accepted: 11 May 2017

Published online: 22 May 2017

\section{References}

Bergelson J, Purrington CB (1996) Surveying Patterns in the Cost of Resistance in Plants. Am Nat 148(3):536-558

Boller T, Felix G (2009) A renaissance of elicitors: perception of microbeassociated molecular patterns and danger signals by pattern-recognition receptors. Annu Rev Plant Biol 60:379-406. doi:10.1146/annurev.arplant.57. 032905.105346

Chen F, Yuan Y, Li Q, He Z (2007) Proteomic analysis of rice plasma membrane reveals proteins involved in early defense response to bacterial blight. Proteomics 7(9):1529-1539. doi:10.1002/pmic.200500765

Chen X, Chern M, Canlas PE, Jiang C, Ruan D, Cao P, Ronald PC (2010) A conserved threonine residue in the juxtamembrane domain of the XA21 pattern recognition receptor is critical for kinase autophosphorylation and XA21-mediated immunity. J Biol Chem 285(14):10454-10463. doi:10.1074/jbc. M109.093427

Chen X, Zuo S, Schwessinger B, Chern M, Canlas PE, Ruan D, Zhou X, Wang J, Daudi A, Petzold CJ, Heazlewood JL, Ronald PC (2014) An XA21-associated kinase (OsSERK2) regulates immunity mediated by the XA21 and XA3 immune receptors. Mol Plant 7(5):874-892. doi:10.1093/mp/ssu003

Chern M, Canlas PE, Fitzgerald HA, Ronald PC (2005) Rice NRR, a negative regulator of disease resistance, interacts with Arabidopsis NPR1 and rice NH1. Plant J 43(5):623-635. doi:10.1111/j.1365-313X.2005.02485.x

Chinchilla D, Zipfel C, Robatzek S, Kemmerling B, Nurnberger T, Jones JD, Felix G, Boller T (2007) A flagellin-induced complex of the receptor FLS2 and BAK1 initiates plant defence. Nature 448(7152):497-500. doi:10.1038/nature05999

Choi HW, Kim YJ, Hwang BK (2011) The hypersensitive induced reaction and leucine-rich repeat proteins regulate plant cell death associated with disease and plant immunity. Mol Plant Microbe Interact 24(1):68-78. doi:10.1094/ MPMI-02-10-0030

Choi DS, Hwang IS, Hwang BK (2012) Requirement of the cytosolic interaction between PATHOGENESIS-RELATED PROTEIN10 and LEUCINE-RICH REPEAT PROTEIN1 for cell death and defense signaling in pepper. Plant Cell 24(4): 1675-1690. doi:10.1105/tpc.112.095869

Choi HW, Kim DS, Kim NH, Jung HW, Ham JH, Hwang BK (2013) Xanthomonas filamentous hemagglutinin-like protein Fha1 interacts with pepper hypersensitive-induced reaction protein CaHIR1 and functions as a virulence factor in host plants. Mol Plant Microbe Interact 26(12):1441-1454. doi:10.1094/MPMI-07-13-0204-R

Huot B, Yao J, Montgomery BL, He SY (2014) Growth-defense tradeoffs in plants: a balancing act to optimize fitness. Mol Plant 7(8):1267-1287. doi:10.1093/ $\mathrm{mp} / \mathrm{ssu} 049$

Hwang IS, du Choi S, Kim NH, Kim DS, Hwang BK (2014) Pathogenesis-related protein $4 \mathrm{~b}$ interacts with leucine-rich repeat protein 1 to suppress PR4btriggered cell death and defense response in pepper. Plant J 77(4):521-533. doi:10.1111/tpj.12400

Jung HW, Hwang BK (2007) The leucine-rich repeat (LRR) protein, CaLRR1, interacts with the hypersensitive induced reaction (HIR) protein, CaHIR1, and suppresses cell death induced by the CaHIR1 protein. Mol Plant Pathol 8(4):503-514. doi:10.1111/j.1364-3703.2007.00410.x

Jung EH, Jung HW, Lee SC, Han SW, Heu S, Hwang BK (2004) Identification of a novel pathogen-induced gene encoding a leucine-rich repeat protein expressed in phloem cells of Capsicum annuum. Biochim Biophys Acta 1676(3):211-222. doi:10.1016/S0167-4781(03)00120-9

Kampinga HH, Craig EA (2010) The HSP70 chaperone machinery: J proteins as drivers of functional specificity. Nat Rev Mol Cell Biol 11(8):579-592. doi:10. 1038/nrm2941

Kearse M, Moir R, Wilson A, Stones-Havas S, Cheung M, Sturrock S, Buxton S, Cooper A, Markowitz S, Duran C, Thierer T, Ashton B, Mentjies P, Drummond A (2012) Geneious Basic: an integrated and extendable desktop software platform for the organization and analysis of sequence data. Bioinformatics 28(12):1647-1649

Kolade OO, Bamford VA, Ancillo Anton G, Jones JD, Vera P. Hemmings AM (2006) In vitro characterization of the cysteine-rich capping domains in a plant 
leucine rich repeat protein. Biochim Biophys Acta 1764(6):1043-1053. doi:10 1016/j.bbapap.2006.03.014

Lemmon SK (2001) Clathrin uncoating: Auxilin comes to life. Curr Biol 11(2):R49R52

McAndrew R, Pruitt RN, Kamita SG, Pereira JH, Majumdar D, Hammock BD, Adams PD, Ronald PC (2014) Structure of the OsSERK2 leucine-rich repeat extracellular domain. Acta Crystallogr D Biol Crystallogr 70(Pt 11):3080-3086. doi:10.1107/S1399004714021178

Miki D, Shimamoto K (2004) Simple RNAi vectors for stable and transient suppression of gene function in rice. Plant Cell Physiol 45(4):490-495

Ogle DH (2017) FSA: Fisheries Stock Analysis. R package version 0.8.12, 0812th edn

Park CJ, Bart R, Chern M, Canlas PE, Bai W, Ronald PC (2010) Overexpression of the endoplasmic reticulum chaperone BiP3 regulates XA21-mediated innate immunity in rice. PLoS One 5(2):e9262. doi:10.1371/journal.pone.0009262

Park YH, Choi C, Park EM, Kim HS, Park HJ, Bae SC, Ahn I, Kim MG, Park SR, Hwang DJ (2012) Over-expression of rice leucine-rich repeat protein results in activation of defense response, thereby enhancing resistance to bacterial soft rot in Chinese cabbage. Plant Cell Rep 31(10):1845-1850. doi:10.1007/ s00299-012-1298-9

Pruitt RN, Schwessinger B, Joe A, Thomas N, Liu F, Albert M, Robinson MR, Chan L, Luu DD, Chen H, Bahar O, Daudi A, De Vleesschauwer D, Caddell D, Zhang W, Zhao X, Li X, Heazlewood JL, Ruan D, Majumder D, Chern M, Kalbacher H, Midha S, Patil PB, Sonti RV, Petzold CJ, Liu CC, Brodbelt JS, Felix G, Ronald PC (2015) The rice immune receptor XA21 recognizes a tyrosinesulfated protein from a Gram-negative bacterium. Sci Adv 1(6):e1500245. doi:10.1126/sciadv.1500245

Pyrzynska B, Pilecka I, Miaczynska M (2009) Endocytic proteins in the regulation of nuclear signaling, transcription and tumorigenesis. Mol Oncol 3(4):321-338. doi:10.1016/j.molonc.2009.06.001

Seo YS, Chern M, Bartley LE, Han M, Jung KH, Lee I, Walia H, Richter T, Xu X, Cao P, Bai W, Ramanan R, Amonpant F, Arul L, Canlas PE, Ruan R, Park CJ, Chen X, Hwang S, Jeon JS, Ronald PC (2011) Towards establishment of a rice stress response interactome. PLoS Genet 7(4):e1002020. doi:10.1371/journal.pgen.1002020

Team RC (2016) R: A language and environment for statistical computing, 332nd edn. R Foundation for Statistical Computing, Vienna

Tian D, Traw MB, Chen JQ, Kreitman M, Bergelson J (2003) Fitness costs of Rgene-mediated resistance in Arabidopsis thaliana. Nature 423(6935):74-77. doi:10.1038/nature01588

Zhang M, Gaschen B, Blay W, Foley B, Haigwood N, Kuiken C, Korber B (2004) Tracking global patterns of $\mathrm{N}$-linked glycosylation site variation in highly variable viral glycoproteins: HIV, SIV, and HCV envelopes and influenza hemagglutinin. Glycobiology 14(12):1229-1246. doi:10.1093/glycob/cwh106

Zhou L, Cheung MY, Zhang Q, Lei CL, Zhang SH, Sun SS, Lam HM (2009) A novel simple extracellular leucine-rich repeat (eLRR) domain protein from rice (OsLRR1) enters the endosomal pathway and interacts with the hypersensitive-induced reaction protein 1 (OsHIR1). Plant Cell Environ 32(12): 1804-1820. doi:10.1111/j.1365-3040.2009.02039.x

Zhou L, Cheung MY, Li MW, Fu Y, Sun Z, Sun SM, Lam HM (2010) Rice hypersensitive induced reaction protein 1 (OsHIR1) associates with plasma membrane and triggers hypersensitive cell death. BMC Plant Biol 10:290. doi:10.1186/1471-2229-10-290

Zhou X, Wang J, Peng C, Zhu X, Yin J, Li W, He M, Wang J, Chern M, Yuan C, Wu W, Ma W, Qin P, Ma B, Wu X, Li S, Ronald P, Chen X (2016) Four receptor-like cytoplasmic kinases regulate development and immunity in rice. Plant Cell Environ 39(6):1381-1392. doi:10.1111/pce.12696

Zipfel C, Kunze G, Chinchilla D, Caniard A, Jones JD, Boller T, Felix G (2006) Perception of the bacterial PAMP EF-Tu by the receptor EFR restricts Agrobacterium-mediated transformation. Cell 125(4):749-760. doi:10.1016/j. cell.2006.03.037

\section{Submit your manuscript to a SpringerOpen ${ }^{\circ}$ journal and benefit from:}

- Convenient online submission

- Rigorous peer review

- Open access: articles freely available online

- High visibility within the field

- Retaining the copyright to your article

Submit your next manuscript at $\gg$ springeropen.com 